\title{
Laintervención pedagógica del profesorado de educación física en un contexto multicultural: prácticas, reflexiones y orientaciones \\ The pedagogical intervention of physical education teachers in a multicultural context: practices, reflections and orientations

\author{
Gonzalo Flores Aguilar*, Maria Prat Grau**, Susanna Soler Prat*** \\ *Universidad de Barcelona (España), ** Universidad Autónoma de Barcelona (España), ***Inefc de Barcelona (España)
}

Resumen. Este trabajo pretende conocer cómo es la intervención pedagógica del profesorado de Educación Física con más de cinco años de experiencia en un conjunto de escuelas de educación primaria con más del 50\% de estudiantes de origen extranjero. Las técnicas para la obtención de los resultados han sido la entrevista semiestructurada y la observación no participante. Para el análisis de datos se ha llevado a cabo el análisis de contenido. De los resultados extraídos se desprende una tipificación teórica de los perfiles pedagógicos del profesorado de Educación Física con experiencia ante la diversidad cultural en sus aulas en función de la intervención pedagógica analizada, además de un conjunto de orientaciones pedagógicas, a nivel personal, curricular y didáctico, encaminadas para el avance hacia una Educación Física intercultural.

Palabras clave. Educación Intercultural; Educación Física; Escuela multicultural; Intervención pedagógica; Profesorado de Educación Física.

Abstract. This paper considers the nature of the pedagogical intervention of PE teachers with over five years' experience in primary schools with more than 50 per cent of foreigner students. Techniques for obtaining the results were the semi-structured interview and non-participant observation, complemented with data content analysis. The results allow us to propose a set of theoretical profiles of physical education teachers with regard to cultural diversity in their classrooms based on the pedagogical intervention analyzed. Finally, we add several teaching guidelines regarding to personal, curriculum and instructional aspects to advance towards intercultural physical education.

Keywords. Intercultural education, Physical education, Multicultural school, Pedagogical intervention, Physical education teacher.

\section{Introducción}

El aumento progresivo de la diversificación cultural del alumnado ha desencadenado un intenso debate acerca del papel de la educación y la escuela a la hora de «promover la cohesión social y el aprendizaje de la vida en comunidad» (García Fernández \& Goenechea, 2009, p.145). Más allá de los primitivos enfoques educativos basados en principios etnocéntricos y relativistas, y aunque estos aún puedan estar presentes (Flecha, 2001; Garreta, 2011), la educación de la ciudadanía del siglo XXI se sitúa inevitablemente en clave intercultural (Essomba, 2008).

A pesar de la ausencia de quórum respecto al corpus científicoteórico de la Educación Intercultural (EI) (Aguado, 2003), esta ha tratado de ser definida y analizada en numerosos trabajos. Una de las definiciones de referencia es la elaborada por Aguado et al. (1999):

Es un enfoque educativo basado en el respeto y la valoración de la diversidad cultural, dirigido a todos y cada uno de los miembros de la sociedad en su conjunto, que propone un modelo de intervención formal e informal, holístico, integrado, configurador de todas las dimensiones del proceso educativo en orden a lograr la igualdad de oportunidades/resultados, la superación del racismo en sus diversas manifestaciones, la comunicación y competencia interculturales (p.40).

Para la adopción y consolidación de los propósitos interculturales en la escuela, el profesorado se concibe como uno de los principales agentes influyentes (Sales, 2007). No obstante, son muchos los estudios que alertan de sus tradicionales déficits formativos al respecto (Besalú, 2004; Etxeberría, Karrera \& Murua, 2010; Flores, Prat \& Soler, 2014).

Respecto ala traducción práctica-pedagógica dela teoría intercultural, García-Fernández y Goenechea (2009) subrayan la visión de la EI como una tautología. Es decir, la EI no conlleva la adopción de un nuevo modelo pedagógico puesto que toda acción educativa debe atender a la diversidad del alumnado. De esta misma manera, Lluch y Salinas (1996) recuerdan que la EI no dispone de unos principios metodológicos ni unas estrategias didácticas específicas ni concretas. En esta línea, Besalú (2002, p.242) opina que «no se trata de inventar nada, sino de recrear la mejor tradición pedagógica, aquella que siempre ha tenido claro que para educar a las personas hay que conocerlas, respetarlas y acogerlas en su diversidad».

Fecha recepción: 12-12-14- Fecha envío revisores: 12-12-14- Fecha de aceptación: 21-04-15 Gonzalo Flores Aguilar gonzalo.flores@ub.edu
Ante este realidad, y de manera más concreta, el profesorado de Educación Física (EF) adquiere un especial interés, sobre todo si se tiene en cuenta el potencial de la EF a la hora de abordar la EI y atender al alumnado de origen extranjero (Capllonch, Godall \& Lleixà, 2007; Soler, Flores \& Prat, 2012). Siguiendo a Flores (2013), la EF posee una serie de ventajas que puede facilitar tal fin (su carácter lúdico y vivencial, su alto componente motivacional, su capacidad de promover relaciones interpersonales, su idoneidad para fomentar la educación en valores, etc.), siempre y cuando el profesorado también actúe para minimizar algunas de sus principales limitaciones (su tradición histórica, el choque de valores corporales y culturales entre el alumnado, los contravalores del área, etc.).

Más allá del discurso teórico, sin embargo, la diversidad cultural genera en las aulas un contexto didáctico específico que es necesario analizar y comprender para poder actuar de la mejor forma posible. Ante el desconocimiento de «muchos aspectos de la didáctica de la EF en entornos multiculturales» (Lleixà, 2002, p.109), en este estudio se plantea la siguiente cuestión: ¿cómo es la intervención pedagógica del profesorado de EF en contextos de gran diversidad cultural?

Para poder abordarla, este trabajo le «da la voz» al propio profesorado de EF ya que es quién vive y lleva a cabo su trabajo diario con alumnado de diversas procedencias en sus escuelas. A partir de su bagaje y experiencia, aquí se recogen los elementos más relevantes de su intervención pedagógica a la vez que se analizan desde el prisma del modelo de EI.

\section{Metodología}

Para la realización de la investigación se ha llevado a cabo un estudio de casos. Así pues, este trabajo se enmarca dentro de una perspectiva interpretativa, puesto que pretende centrarse en la comprensión de los fenómenos educativos a partir de los significados e intenciones de las personas implicadas en el contexto educativo (Latorre, Del Rincón \& Arnal, 2003). En este caso, a partir del profesorado.

\section{Participantes}

Para la elección de los casos de estudio se ha realizado un muestreo intencional o de casos típicos (Heinemann, 2003), seleccionándose a doce docentes (cinco hombres y siete mujeres) con más de cinco años de experiencia laboral en escuelas públicas de educación primaria con más del 50\% de alumnado de origen extranjero, en su mayoría dentro de la ciudad de Barcelona (ver tabla 1). 
Tabla 1.

Características del profesorado participante relativas al curso 2010-2011. Elaboración propia.

\begin{tabular}{ccccc}
\hline Escuela/Ubicación & Pseudónimos & Sexo & Edad & Años de experiencia \\
\hline A. Sant Martí (Barcelona) & Laia & M & 54 & 13 \\
\hline A. Sant Martí (Barcelona) & Darío & H & 31 & 6 \\
\hline B. Ciutat Vella (Barcelona) & Rodrigo & H & 43 & 17 \\
\hline C. Ciutat Vella (Barcelona) & Natalia & M & 36 & 6 \\
\hline C. Ciutat Vella (Barcelona) & Carmen & M & 43 & 12 \\
\hline D. Ciutat Vella (Barcelona) & Lorena & M & 33 & 5 \\
\hline E. Sants-Montjüic (Barcelona) & Mar & M & 43 & 13 \\
\hline F. Sants-Montjüic (Barcelona) & Ariadna & M & 34 & 7 \\
\hline G. Sant Andreu (Barcelona) & Hugo & H & 39 & 7 \\
\hline H. Nou Barris (Barcelona) & Álvaro & H & 34 & 9 \\
\hline I. Gràcia (Barcelona) & Lucas & H & 56 & 15 \\
\hline J. Sabadell & Mireia & M & 45 & 11 \\
\hline
\end{tabular}

La elección del profesorado con una amplia experiencia profesional en la misma escuela multicultural queda justificada por el especial interés en profundizar en la evolución de la trayectoria profesional de este colectivo, además de sus conocimientos y saberes. En cuanto al porcentaje de estudiantes de origen extranjero, este trabajo pretende adentrarse en escuelas donde la realidad multicultural está más presente, ya que el general desconocimiento de su «día a día» provoca normalmente la estigmatización de estos centros y alumnos/as.

\section{Instrumentos}

La primera técnica de recogida de datos ha sido la entrevista semiestructurada, la cual se caracteriza por la flexibilidad en la formulación de las preguntas, en su mayoría de carácter abierto, que aparecen plasmadas en el guión elaborado previamente (Massot, Dorio \& Sabariego, 2004). La observación no participante ha sido la segunda técnica empleada, ya que el observador se ha mantenido al margen para interferir lo menos posible en las sesiones observadas (Heinemann, 2003). A su vez, las notas de campo se han utilizado como registro narrativo de lo observado, sobre todo debido a la poca rigidez y flexibilidad de las mismas (Mckernan, 1999).

Para la codificación, el análisis y la interpretación de los resultados se ha realizado un análisis de contenido siguiendo las fases establecidas por Bardin (1986). Para ello se ha recurrido al apoyo del programa informático «Nudist NVivo®» (versión octava).

\section{Análisis de datos}

A partir de un proceso deductivo-inductivo, los elementos de la intervención pedagógica del profesorado que se han considerado más relevantes para el análisis ante la realidad multicultural se agrupan entorno a dos grandes aspectos: el planteamiento curricular establecido inicialmente y el enfoque didáctico en el aula realizado de forma cotidiana, por la otra. Dentro de estos, los indicadores correspondientes se aprecian en la siguiente tabla 2.

Tabla 2 .

Variables e indicadores del estudio. Elaboración propia.

\begin{tabular}{cl}
\multicolumn{1}{c}{ Variables } & \multicolumn{1}{c}{ Indicadores } \\
\hline \multirow{3}{*}{ Planteamiento curricular } & Programación curricular \\
& Adaptaciones curriculares \\
& Sistemas de evaluación \\
\hline \multirow{3}{*}{ Enfoque didáctico en el aula } & Sistema de acogida \\
& Estrategias comunicativas \\
& Formación de grupos \\
& Estrategias motivadoras \\
& Relación profesorado-al umnado \\
\hline
\end{tabular}

En cuanto a la posterior presentación de los resultados, en este trabajo se utilizan dos tipos de abreviaturas para identificar la fuente de origen de los mismos. Los fragmentos procedentes de la entrevista poseen la letra E (ejemplo: E-Darío) y los relativos a las notas de campo las letras NC (ejemplo: NC-Darío).

\section{Resultados y discusión}

Los resultados que a continuación se presentan y discuten aparecen estructurados en los siguientes cuatro apartados: a) planteamiento curricular; b) enfoque didáctico; c) perfiles pedagógicos del profesorado de EF; y d) orientaciones pedagógicas para una EF intercultural.

\section{a) Planteamiento curricular}

La programación curricular: sin cambios, pero aplicada con flexibilidad e incorporando puntualmente los «juegos del mundo»

La totalidad del profesorado participante muestra un especial interés a la hora de subrayar que la presencia del alumnado de origen extranjero no conlleva necesariamente ninguna modificación o revisión al respecto, independientemente de sus características y particularidades.

En EF claro que hay cambios, pero por diversidad cultural la programación general la cambias poco (E-Dario).

Cambiar programaciones o maneras de hacer porque haya multiculturalidad, aquíno(E-Mireia).

En cambio, López-Rodríguez y Sentís (2000) revelan que toda programación curricular debería ser acorde con las posibles necesidades educativas que proceden de la diversidad del alumnado (capacidades cognitivas, estilos de aprendizaje, procedencias culturales, intereses, etc.).

Por otro lado, Lucas, Hugo y Lorena confirman que, ante la imprevisibilidad del día a día, es necesario improvisar. Por este motivo, estos docentes destacan la poca rigidez de sus programaciones curriculares no cerradas.

Por mi forma de ser, quizás, a mí me resulta muy difícil limitarme a una programación concreta. Puede ser que tenga programado

hacer algo y aquel día, por lo que sea, pues vamos a hacer otra cosa que no tiene nada que ver con lo que estaba programado. A veces sale mejor que lo que hubieras tenido programado, y a veces pues tampoco sale bien (E-Lucas).

Como señala Lleixà (2002), la flexibilidad curricular es un aspecto destacable entre el discurso del profesorado deEF que trabaja en contextos multiculturales. Aún así, sería importante diferenciar a la ocasional flexibilización de lo previamente programado de la improvisación diaria descontextualizada, ya que detrás de esta última manera de actuar pueden encontrarse docentes desmotivados y prácticas poco educativas (Flores, 2013).

Sobre este tema, Lorena tiene una opinión más crítica respecto a la viabilidad de las programaciones curriculares.

Yo no creo en la programación, creo más en las memorias finales. ¿De qué te vale tenerlo todo pensado y cuadriculado? Cuando vienen los días raros o torcidos la programación no vale para nada, tienes que improvisar y saber actuar (NC-Lorena).

Aunque el profesorado afirme no haber modificado en gran medida su programación curricular, diversos participantes sí que destacan la introducción de algunas unidades didácticas de juegos del mundo a raíz de la diversificación cultural de su alumnado.

Añadí una unidad de programación sobre juegos interculturales, que no es una adaptación curricular. Entonces cada chaval le preguntaba a sus padres juegos de su niñez (E-Álvaro).

Este año he hecho con los de ciclo superior una unidad de juegos de alrededor del mundo. Estuvimos trabajando con los cinco continentes (...) hicimos una búsqueda de juegos de sus orígenes. La verdad es que fue súper-interesante, y los juegos eran súper-divertidos, les encantó (E-Ariadna).

Este hecho se concibe indispensable dentro de la EI, ya que la realización de estas unidades didácticas pueden permitir que el profesorado tenga en cuenta previamente los referentes culturales y las experiencias del alumnado, para así favorecer el descubrimiento de elementos universales y la valoración de las diferentes aportaciones culturales (Banks, 1995; Gieß-Stüber, 2009).

Sin embargo, en los resultados no se han encontrado las suficientes evidencias que confirmen si dichas prácticas van más allá de los típicos proyectos puntuales, en forma de anécdota o moda pasajera.

Sobre esta idea, cabría destacar las palabras de Rodrigo:

En algunos momentos, los primeros días sí que hacemos algún juego de su país [del alumnado de origen extranjero], que a veces conocen y a veces no. Les ayudamos un poco a integrarse (ERodrigo). 
En este caso, el hecho de que el alumnado de origen extranjero no conozca los juegos del mundo que en teoría pertenecen a su país podría convertirse en un arma de doble filo. Por una lado, el propio alumnado puede sentirse frustrado culturalmente, ya que se le presupone un conocimiento lúdico, en este caso folclórico y estereotipado. Por otra parte, la simple práctica de un juego etiquetado como propio de un país en concreto no implica per se la facilitación de la integración del alumno recién llegado, ya que otros muchos aspectos emocionales y sociales se deben tener en cuenta.

En definitiva, el profesorado de EF debería ser consciente que este tipo de prácticas permiten la construcción superficial y trivial de un currículum intercultural reproductor de diferentes estereotipos culturales (Fecha \& Puigvert, 2002; Torres, 2008).

Las adaptaciones curriculares: la condición de inmigrante no implica la necesidad de adaptar, pero a veces se hace sin decirlo

Una gran mayoría del profesorado afirma que la presencia de alumnado de origen extranjero no suele implicar la necesidad de llevar a cabo ningún tipo de adaptación curricular en el área de EF, ya que estos estudiantes «lo pueden hacer todo también» (E-Ariadna) o «casi nunca hay que realizarlas [adaptaciones curriculares], y mucho menos por el simple hecho de que un alumno sea o no inmigrante (...). La naturaleza de su asignatura ayuda a no tener que adaptar nada» (NC-Lorena).

Además de coincidir con los resultados del trabajo de Pastor, González, Cuevas y Gil-Madrona (2010), esta manera de actuar contradice a las orientaciones de Lleixà (2002) al respecto: las adaptaciones curriculares son esenciales para fomentar entre el alumnado, en base a sus principales características y necesidades, la igualdad de oportunidades.

En cambio, las adaptaciones curriculares que normalmente sí que se aplican suelen destinarse al alumnado que presenta algún tipo de discapacidad, intelectual o física, independientemente de su origen cultural.

Adaptaciones curriculares no, porque normalmente hacen lo que hacen los demás [alumnado de origen extranjero]. Lo único que hacemos de adaptaciones es si tienen algún problema físico, que tengas algún ciego, algún sordo (E-Mar).

Este tipo de discursos puede hacer pensar que algunas de las necesidades propias del alumnado de origen extranjero (estado emocional, choque cultural, etc.) a menudo pasan desapercibidas para el profesorado de EF. Como señalan Aguado (2006) y Gil-Jaurena (2008), aquí las diferencias culturales tampoco se consideran tan relevantes como las diferencias motoras o perceptivas.

Por tanto, podríamos hablar de la existencia de un profesorado que no se cuestiona el carácter homogeneizador y etnocéntrico del currículum y de sus actuaciones (Garreta, 2011; Ortiz, 2008). Este desconocimiento contribuye, así, a las resistencias al cambio a la hora de modificar o adaptar las actuaciones educativas en un contexto multicultural (LópezPastor, Pérez, \& Monjas, 2007; Oliver, 2003).

No obstante, aunque explícitamente el profesorado puede señalar que no se hacen adaptaciones curriculares, o no las haya etiquetado o percibido así, se observa como en la práctica sí que lleva a cabo actuaciones que se podrían considerar adaptaciones curriculares, algo que también ocurre entre el profesorado estudiado en Capllonch et al. (2007). Más concretamente, estas hacen referencia a la atención del alumnado musulmán que realiza el Ramadán (contenidos menos físicos, más pausas para descansar, etc.).

El alumnado que puedeya hace el Ramadán, entonces ya séque en aquellas semanas no les hago actividades de mucha carga física (E-Rodrigo).

Ahora el Ramadán está cayendo en verano. Entonces les digo: «imira! cuando te canses lo dejas y ya está» [...] «si tú ves que no puedes no lo haces y ya está» (E-Mireia).

Este tipo de adaptaciones, aunque otra vez hacen referencia a aspectos «físicos» y no a aspectos emocionales o culturales, coinciden con las llevadas a cabo por Carroll y Hollinshead (1993) y Dagkas (2007).
Los sistemas de evaluación: entre el sistema tradicional y la valoración de las actitudes

Los sistemas de evaluación a los que recurre el profesorado de este estudio se enmarcan en dos perspectivas diferentes.

Por un lado, una parte del profesorado reproduce la evaluación tradicional de la EF basada en la medición de resultados físicos mediante una batería de pruebas físicas. López-Pastor (2007) y Lleixà (2002) son algunos de sus detractores. Como señala Lleixà (2002), esta evaluación es incompatible con una EF respetuosa hacia la diversidad y hacia la «especificidad de cada persona y su nivel de realizaciones en el ámbito de la actividad motriz» (p.107).

Contrariamente a esta idea, una gran mayoría del profesorado identifica a la dimensión actitudinal del alumnado como el criterio más influyente y relevante en la evaluación de la asignatura. Más allá del nivel de habilidad motriz que finalmente alcanza el alumnado, algunos de los criterios de evaluación más importantes son: a) la actitud de respeto y la tolerancia hacia los compañeros y compañeras y el profesor o profesora; b) el grado de implicación e interés por la asignatura; c) el buen comportamiento disciplinario en la clase; y d) el cumplimiento de las normas higiénicas (chándal, ropa deportiva).

Mi evaluación se centra prácticamente, en un 99\%, en la actitud: la actitud de respeto, que hagan la clase, que haya ilusión, que intenten divertirse, que no creen problemas, más de que si me hacen bien o mal las actividades (E-Lorena).

Por tanto, este último grupo de docentes hacen valer las sugerencias de Contreras (2002) y Lleixà (2002) al respecto: la evaluación de la dimensión actitudinal del alumnado cobra una especial relevancia e interés en un aula multicultural.

\section{b) Enfoque didáctico}

Acogida y comunicación en las clases de Educación Física: por sí sola o con atención específica

El profesorado aborda la acogida del alumnado recién llegado de dos maneras diferentes.

Existe un primer grupo de docentes, poco numeroso, que no realiza ningún tipo de actuación concreta. Según este colectivo, la EF posee una serie de ventajas que permiten no crear ningún protocolo específico de actuación para facilitar la acogida del nuevo estudiante, puesto que son suficientes las dinámicas que se crean en clase.

Establecer un protocolo en EF de acogida pues no [...]. La acogi-

da, por la misma idiosincrasia de la asignatura, hace que no sea muy necesaria (E-Lucas).

¿Podría significar esto la existencia de un profesorado despreocupado por la acogida del alumnado? o, ¿sería esta una manera de dar normalidad y naturalidad a las personas que acaban de llegar, tal y como aconsejan Oller y Colomé (2010)?

Independientemente de las respuestas a estas preguntas, es importante resaltar que el alumnado recién llegado puede sentir en sus inicios de escolarización una serie de necesidades, sobre todo psicológicas (Besalú \& Tort, 2009), que el profesorado debería tener en cuenta. Por ello, pensamos que el hecho de no hacer nada al respecto puede facilitar el aislamiento y la inseguridad inicial del nuevo estudiante.

En el lado contrario, en este trabajo se identifican otro grupo de docentes que sí que se preocupan específicamente por facilitar la acogida del nuevo alumnado. Entre las estrategias de acogida encontradas se destacan: a) el conocimiento previo del nuevo alumnado antes de que acuda por primera vez a la clase de EF; b) una postura protectora durante las sesiones mostrándose cercano al estudiante, para darle confianza; c) la realización de diferentes actividades de presentación; y d) las estrategias comunicativas utilizadas para disminuir dificultades lingüísticas, como el aprendizaje de otros idiomas (inglés y francés) o el de ciertas palabras en idiomas como el tagalo, urdú, chino, etc., y como la asignación de compañeros y compañeras traductores e intérpretes.

El primer día que llegan a la clase yo ya intento entrar en el aula de acogida, conocerles antes de que vengan a la clase. Presentarme, hablar con ellos un poquito, como están (...). A mí me gusta siem- 
pre un poco el hablar con ellos personalmente para que también pierdan un poco el miedo, que cojan la confianza (E-Álvaro). Normalmente hay una presentación, pues conocer de donde es [...]. Es crear un ambiente para hacer una acogida más cercanay que la distancia no sea tan evidente. Bueno, le preguntas de qué país viene, te presentas tú como profesora (E-Ariadna).

Previamente yo ya he aprendido las palabras agradables [en otros idiomas]. Palabras clave para relacionarme con él (E-Hugo).

Cabe recordar que buena parte del éxito del enfoque intercultural y del futuro educativo del alumnado recién llegado pasa por el desarrollo de una adecuada acogida (Carbonell, 2006; Oller \& Colomé, 2010).

En cuanto a las estrategias comunicativas, la figura del compañero o compañera traductor e intérprete es la principal estrategia lingüística a la que recurre el profesorado de EF en su aula.

Si hay alguno en el grupo clase que conozca el idioma del alumnado recién llegado, para favorecer la acogida se puede contar con alumnado intérprete (E-Rodrigo).

Mientras los alumnos están jugando, veo como una de las alumnas recién llegadas va siempre junto a otra. Según la profesora, es su «compañera intérprete». En los casos que sean necesarios, le ayuda a entender los ejercicios, le explica lo que dicen sus compañeros, y le sirve como medio para poder comunicarse con el resto de alumnos y con la profesora (NC-Lorena).

Aunque en algunos casos su presencia tenga una duración determinada, puesto que el profesorado piensa que su prolongación en el tiempo provoca un retroceso en el aprendizaje del catalán, esta se concibe como una medida indispensable y factible, sobre todo debido al alto grado de accesibilidad.

Intentamos que los niños hablen catalány castellano, porque cuando se encuentran fuera (...) si tú hablas pakistaní y no hablas nada [catalány/o castellano], ¿cómovas a poder comunicarteoexpresarte en el momento que tú tengas alguna dificultad? (E-Natalia).

Sobre este tema, los trabajos de Carrasco (2006) y López-Pastor et al. (2007) también hacen referencia a la utilización de esta figura en las clases de EF. De hecho, García-Fernández y Goenechea (2009) subrayan que esta práctica tiene efectos muy positivos, tanto en la persona tutelada, como en la persona que ejerce el rol de tutor/a.

Otra de las estrategias comúnmente más utilizadas por el profesorado consiste en la visualización e imitación de las demostraciones prácticas de los ejercicios. En este sentido, Hugo afirma que «con la percepción visual automáticamente el alumnado ya entiende lo que hay que hacer, y lo hace» (E-Hugo). De igual modo, Álvaro considera que la «EF es muy intuitiva» y por ello, en sus clases se puede llevar a cabo la «imitación visual». Es decir, «siempre intentar enseñar visualmente cómo se hace el ejercicio» (E-Álvaro).

En esta línea, Mireia y Laia opinan lo siguiente:

Yo es que lo tengo muy fácil porque aunque no me entiendan, como en EF es todo bastante visual, pues en principio no hay ningún problema (E-Mireia).

La EF es el área más perfecta en ese sentido, para la imitación y para la mímica. A los tres días no te das cuenta de que hay un niño recién llegado, para nada (E-Laia).

A pesar de la importancia que adquiere la información visual, se han detectado casos donde el alumnado tiene ciertas dificultades para entender las demostraciones realizadas.

Hay niños que son más introvertidos y les da más vergüenza (...) hay niños que ni por imitación y que quizás llevan aquí tres o cuatro meses y aún están perdidos (E-Natalia).

De esta manera podría cuestionarse la supuesta poca relevancia del conocimiento del idioma vehicular de la escuela en las clases de EF, tal y como destacan algunos participantes:

Los chavales que vienen de fuera quizás no entenderán el idioma, pero tontos normalmente no son, y tienen ojos. Ven lo que hacen los demás e imitan (E-Lucas).

Aunque no me entienda, como en EF todo es bastante visual, pues en principio no hay ningún problema (E-Mireia)

Estos últimos argumentos coinciden con los de Contreras, Gil,
Cecchini y García (2007) y Gil-Madrona y Pastor (2003) a la hora de considerar que el lenguaje verbal no es un aspecto limitador para el alumnado de origen extranjero en las clases de EF.

En tercer lugar, en este estudio también se revela la presencia de un reducido número de participantes que muy asiduamente recurren al lenguaje corporal (mímica, gestos, etc.) como medio de comunicación para facilitar la comprensión verbal.

Cuando llega un alumno nuevo (...) todo con gesticulación porque claro, yo pakistaní no sé, y chino tampoco (E-Natalia).

Cuando Lorena se dirige a su alumnado utiliza un tono de voz no muy alto y un ritmo de voz pausado. La comunicación verbal se acompaña en todo momento de una gran cantidad de gestos y posturas [lenguaje corporal], es decir, realiza diversas demostraciones corporales para facilitar el entendimiento de sus palabras. Se observa una profesora muy expresiva (NC-Lorena).

Gieß-Stüber y Kühler (2008) ya ofrecieron estas mismas directrices al respecto en sus respectivos trabajos.

Finalmente, y a lo que la dimensión lingüística se refiere, entre el profesorado participante existen nuevamente diferentes maneras de intervenir.

Por un lado, algunos docentes muestran un especial interés en fomentar el catalán como la principal lengua vehicular de la escuela. De hecho, se han encontrado casos donde el profesorado aprovecha su asignatura para mejorar esta competencia lingüística.

Interesa que este alumnado [origen extranjero] tenga unos conocimientos de la lengua catalana, que es la lengua vehicular de la escuela, para evitar la exclusión social (E-Ariadna).

Para facilitarle la introducción al idioma [catalán], yo por ejemplo, cuando trabajo en la pista, trabajo mucho la geometría, trabajo el tema de colores, de numeración, si las pelotas están numeradas, pues puedo trabajar pares e impares (...), todo esto me sirve para irlo introduciendo (E-Laia).

Mientras que Bell y Lorenzi (2004) y Patterson (2012) destacan a las clases de EF como espacios idóneos para el aprendizaje idiomas, sobre todo el vehicular de cada escuela, Etxeberría y Elosegui (2010) alertan del peligro que conlleva el posible el veto lingüístico (prohibir la utilización de los idiomas no oficiales), puesto que existe el riesgo de aislamiento y autoexclusión del alumnado ante el pánico, el miedo o la inseguridad que le puede provocar hablar en un lenguaje que quizás aún desconoce.

Lejos de esto, otros participantes también utilizan el inglés y algunas palabras clave en idiomas minoritarios en sus sesiones para dirigirse al alumnado de origen extranjero.

Como saben un poquito inglés, los filipinos, los indios y los urdus, entonces chapurreas en inglés como puedes (E-Ariadna).

Se ríen mucho porque digo palabras que suenan mal en chino, yo he intentado hacerlo muchas veces en chino y me salen palabras que no (...), y se ríen, pero bueno, me parece una posibilidad que está bien (E-Hugo).

Autores como Goenechea, García-Fernández y Jiménez (2011) y Jordán (2005) consideran muy adecuada esta última estrategia dentro de una escuela multicultural. Según Etxeberría y Elosegui (2010), los estudiantes pueden ver el «guiño» que estos profesionales dedican a sus idiomas maternos, lo cual es un ejemplo de valoración positiva hacia sus culturas, con las ventajas emocionales que esto conlleva para el alumnado.

\section{Las estrategias para la formación de grupos: aleatoria o intencio- nada}

El profesorado de este estudio baraja dos opciones a la hora formar los grupos o parejas de trabajo en el aula: una aleatoria, basada incluso en el azar; y otra intencionada, según unos criterios planificados.

Dentro de la distribución intencionada, el criterio de la nacionalidad del alumnado es el menos utilizado por el profesorado, salvo que sea forzosamente necesario (compañero/a intérprete). Es decir, diversos docentes afirman que agrupar las mismas nacionalidades puede generar una división que podría favorecer el aislamiento y la discriminación entre los diversos grupos culturales. 
Por ejemplo, en sexto me coinciden tres alumnos de la misma nacionalidad. Intento que no vayan juntos para que se esfuercen un poco más en intentar hablar el idioma y que se relacionen (E-Mar). Esta manera de actuar puede favorecer el contacto y la interacción constructiva entre los diversos grupos culturales.

Ellos ya saben que con la gente que están siempre en el patio no pueden estar trabajando en EF. Yo pienso que tienen que aprender a trabajar con diferentes tipos de gente, les guste o no les guste ( $E$ Lucas).

A veces los grupos los hago yo. Aquí lo que intento es trabajar mucho la cohesión social, porque también creo que esta escuela, a nivel de barrio y su situación, requiere un poquito el no excluirse (E-Ariadna).

Sobre este tema, Gieß-Stüber (2000) es una de las autoras que más hincapié hace en la relevancia del contacto y el intercambio entre grupos culturales en el aula.

Aún dentro de las agrupaciones intencionadas, y de manera puntual, algunos docentes también pueden llegar a recurrir al criterio de afinidad (amigos y amigas) como medida para evitar conflictos.

Hugo se dirige a mí para decirme que cuando hay una clase tan problemática agrupa a los alumnos según la afinidad que ellos tengan. De esta manera se evitan que surjan más problemas (NCHugo).

En cuanto a la libre elección, el profesorado permite que esta se lleva a cabo siempre y cuando el alumnado cumpla con una de las normas básicas de distribución: la formación de grupos mixtos en cuanto al sexo del alumnado.

Siempre intento hacerlo mixto, nunca hago chicos y chicas. Aellos sí que les gustaría: «chicos contra chicas», pero siempre es mixto (E-Darío).

Hago grupos mixtos, [...] depende de la actividad, los hago yo directamente, pero normalmente los hacen ellos. Bueno, si yo hago elegir a dos niños, han de escoger niño-niña-niño-niña, y si es niñaal revés(E-Carmen).

\section{Motivar al alumnado, ¿sí o no?}

Una parte del profesorado destaca el alto nivel de motivación que posee la mayoría del alumnado hacia los contenidos de la EF en una escuela multicultural. Por este motivo, estos docentes creen que no es necesario llevar a cabo ninguna estrategia motivadora en clase.

En mi asignatura se motivan solos (E-Mireia).

No es necesario motivar, porque por suerte es un área que motiva a todos: pocos niños y niñas no están motivados a la hora de EF (E-Rodrigo).

Esta percepción también ha sido expresada por el profesorado estudiado en Capllonch et al., (2007) y Ruiz-Valdivia, Molero, Zagalaz y Cachón (2012).

En el lado opuesto, los resultados revelan la existencia de varios participantes que destacan el papel fundamental que adquiere motivar al alumnado durante las clases de EF.

Hay que motivar mucho al alumnado recién llegado y al no recién llegado. La motivación, si no existe, es complicado hacer cualquier cosa, ya sea en clase o en EF (E-Darío).

De hecho, una mínima parte del profesorado reconoce que no todo el alumnado se muestra motivado hacia la EF, especialmente las chicas y el alumnado recién llegado. Por ello, estas personas afirman tener la necesidad de introducir diferentes estrategias motivadoras: retos, premios, expresiones afectivas, cambios de rol, etc.

Tengo niñas que están totalmente desmotivadas y no sé cómo engancharlas, no tienen ganas de hacerlo [ejercicios EF]. Voy detrás de ellas, hablo con las tutoras, hablo con ellas y no, y las castigas y a lo mejor ya te vienen vestidas o te vienen con cosas que sabes que no les vas a dejar hacer clase. Es que no quieren (ELorena).

Observo como la maestra anima y motiva a una de sus alumnas menos hábiles. Cuando una estudiante pakistaní, la cual visiblemente tiene problemas motores y de coordinación, consigue saltar las vallas eficazmente, la profesora le abraza y le felicita públicamente (NCLorena).

Hugo me explica que cada año otorga unos premios y trofeos para todos los alumnos que se lo merecen: «compro copas y medallasy me invento el premio de la deportividad, el del compañerismo, el del mejor velocista, el mejor futbolista, etc.». Finalmente me dice: «también tienen que vivir la sensación de victoria, porque en su día a día viven muchas derrotas» (NC-Hugo).

En esta línea, Prat y Soler (2002, 2003) y Soler (2009) también destacaron la baja motivación que a menudo puede poseer el colectivo femenino hacia la EF. Además, cabría recordar que, junto con la posible percepción de la incompetencia motriz que puede poseer el propio alumnado (Ruiz-Pérez, 2000), los contrastes culturales que pueden existir a nivel del bagaje lúdico y motriz, e incluso el propio lenguaje corporal (Flores, 2013), pueden afectar negativamente al grado de motivación que presenta el alumnado de origen extranjero hacia la EF.

Por todo ello, y en el caso concreto de las clases de EF que acontecen en contextos de diversidad cultural, Dagkas (2007) destaca la importancia que adquiere el énfasis motivacional de la intervención del profesorado de EF, sobre todo a partir de sus retroalimentaciones al alumnado durante y posteriormente a la práctica.

La relación del profesorado de EF con el alumnado: ¿a qué distancia? ... ¿afectuoso o distante?

En función de la clasificación que lleva a cabo Jackson (1968) respecto al tipo de relación que puede mantener el profesorado con su grupo de estudiantes, la información recopilada en el trabajo de campo de este estudio permite identificar, a grandes rasgos, dos estilos de docentes: el afectuoso y el distante.

Dentro del primer grupo se encuentran un conjunto de docentes que presentan un alto grado de preocupación por el bien pedagógico de su alumnado, sobre todo a partir del establecimiento de unas estrategias afectivas que aumentan el grado de confianza entre ambos.

Si ves que el chaval está triste, está apático ante la asignatura o ante el trabajo, pues sí que piensas a ver de qué manera le puedo motivar, o de quémanera puedo hacerle trabajar, a ver si con estos niños que conecta más (...) (E-Álvaro).

Por lo visto, la gran mayoría de niños de esta clase juegan en el equipo de fútbol del barrio, y el profesor acostumbra a ir a verlos jugar junto a sus hijos (NC-Hugo).

En esta línea, en uno de los casos también se hace referencia a la creación de un vínculo afectivo-emocional.

Lo único que quieren realmente estos niños es un poco de afectividad, y con eso ya lo has solucionado todo [...] aquí con los niños tienes un trato muy afectivo, demasiado. A veces haces más de mamá que no de profesora (E-Carmen).

Es como una relación de amigo, pero claro, manteniendo siempre el respeto, pero como de amigo y de cercanía (E-Lorena).

Para que esto sea posible, el mismo profesorado reconoce la necesidad de cambiar sus actitudes, más aún si en ellas existen prejuicios y estereotipos hacia el alumnado.

Yo los quiero un montón [en referencia al alumnado gitano], ese es el primer paso, que yo me quite mi barrera y acepte a la gente como es [...] no puedes llegar y estar etiquetando (E-Hugo).

Bajo esta misma idea, Jordán (2004) considera que uno de los primeros pasos para la formación intercultural del profesorado, en formación o en activo, consiste en la revisión, concienciación y cambio de las actitudes hacia la diversidad cultural.

Con todo ello, es importante dejar constancia que dentro de este perfil de docentes se han identificado algunas de las actitudes profundas que Van Manen (1998) reclama a todo docente, como son: la responsabilidad, la esperanza y el amor pedagógico, las cuales se insertan dentro del denominado «tacto pedagógico» (Ibíd.).

En el lado opuesto, dentro del profesorado distante, se encuentra el caso de una educadora desinteresada, debido principalmente a sus difíciles experiencias pasadas.

Mar se autodescribe como «una pasota». Según ella: «esto es 
debido a los muchos años de gritos y preocupaciones» (NC-Mar). Mar me dice lo siguiente: «yo soy profesora y no una asistenta social o una monja de la caridad [...] no quiero profundizar en el origen de los problemas de mis alumnos» (NC-Mar).

Llama la atención cómo, aunque los otros participantes también hayan descrito vivencias negativas durante su trayectoria profesional, ninguno de ellos ha justificado ni afirmado abiertamente mantener esta relación problemática con el alumnado, lo cual deja entrever la difícil situación personal y profesional en la que se encuentra Mar. Al igual que ella, una parte del profesorado estudiado en Camacho y Padrón (2006) también alude a la presencia de comportamientos disruptivos en el aula y a la diversidad del alumnado como algunos de los muchos factores influyentes en el malestar docente.

\section{c) Perfiles pedagógicos del profesorado de EF}

Tras analizar el conjunto de actuaciones del profesorado, y en base a la perspectiva teórica de la EI, se considera especialmente útil aportar una tipificación teórica de los perfiles pedagógicos del profesorado de EF con experiencia ante la diversidad cultural en sus aulas. En el estudio de Flores (2013) se describen el resto de rasgos relacionados con la percepción del alumnado de origen extranjero y la trayectoria profesional del profesorado.

Esta propuesta no responde a la descripción de casos reales en los que una sola persona reúna todas las características de un determinado perfil. De hecho, en cada perfil se han agrupado todos aquellos aspectos relacionados con las variables de estudio que se ajustan a un mismo modo de ver y de hacer del profesorado ante la diversidad cultural.

Así pues, en este trabajo se identifican tres tipos de perfiles docentes en función de sus intervenciones pedagógicas: a) el profesorado sensible-inclusor; b) el profesorado romántico-asimilador, y c) el profesorado quemado-prejuicioso.

El profesorado de EF sensible-inclusor se caracteriza por llevar a cabo una intervención que denota preocupación por el bien pedagógico de todo su alumnado. Para ello no duda en realizar adaptaciones curriculares aunque no las denomine así, atender a las necesidades de su alumnado, además de buscar nuevas estrategias de todo tipo de cara a igualar las oportunidades y favorecer la participación de todos y todas en las clases deEF.Además, todo esto se lleva a cabo dentro de un clima de aula marcado por la acogida y los altos niveles de afectividad existentes entre el profesorado y alumnado.

En segundo lugar, las intervenciones pedagógicas del profesorado de EF romántico-asimilador vienen determinadas por su visión utópica de la EF como panacea. Es decir, la EF como asignatura mágica que agrada a todo el alumnado, tal y como es, lo cual quiere decir que no es necesario hacer nada nuevo o especial debido a la presencia del alumnado de origen extranjero. De esta manera, este alumnado vivencia situaciones de aculturación, además de que sus dilemas y necesidades pasan desapercibidas por el profesorado.

En último lugar se encuentra el perfil de profesorado quemadoprejuicioso. La falta de compromiso profesional y las resistencias al cambio de esta parte de los participantes provocan la reproducción de unas prácticas discriminatorias y estereotipadas que el alumnado tiene que padecer, pudiéndose agravar así las iniciales dificultades personales y sociales de este colectivo.

\section{d) Orientaciones pedagógicas para una EF intercultural}

Al igual que en el apartado anterior, de la combinación de los resultados y de la revisión de la literatura, aquí se desprenden un conjunto de orientaciones pedagógicas para el profesorado que se muestre dispuesto a avanzar hacia una EF intercultural.

A nivel de desarrollo personal:

- Fomentar, promover y mantener una conciencia crítica y reflexiva respecto a la realidad social, el papel de la escuela, a las propias actitudes individuales y a la acción educativa que finalmente tiene lugar en el aula.

- Aproximarse, conocer y contrastar distintas realidades cultu- rales que les permitan valorar por igual las diversas culturas, evitando la visión romántica e idealista de cada una de ellas.

A nivel de desarrollo curricular:

- Elaborar las adaptaciones curriculares pertinentes para aquél alumnado que requiera algún tipo de especificidad sin rebajar los objetivos didácticos.

- Seleccionar y desarrollar contenidos de la EF variados, desconocidos o poco habituales, de cara a igualar los niveles de habilidad que inicialmente presenta el alumnado, y así fomentar una igualdad de oportunidades para todos y todas.

- Incorporar de forma progresiva al alumnado recién llegado en aquellas prácticas que pueden suponer un mayor choque cultural, ya sea debido a las diferencias en la cultura corporal o al desconocimiento de las prácticas motrices y lúdicas.

- Recoger el bagaje lúdico y deportivo de las familias de los niños y niñas inmigrantes e incorporarlo en las clases.

- Resaltar las similitudes culturales por encima de las diferencias y poner en evidencia los procesos de «mestizaje», evitando visiones folclóricas y superficiales de las diversas culturas.

- Buscar elementos significativos y motivadores para el alumnado de origen extranjero, que promuevan su implicación y participación: incorporar juegos, danzas, actividades, etc. que ya les resulten familiares; poner referentes de deportistas (de élite o próximos al alumnado) de diversos países.

- Promover una evaluación donde la dimensión actitudinal del alumnado, sobre todo respecto a las actitudes referidas a los valores humanos (solidaridad, respeto, tolerancia, cooperación, etc.), destaque por encima del logro de habilidades y destrezas motrices.

- Apostar por el establecimiento de sistemas de evaluación formativos, donde el alumnado pueda ser partícipe directo del mismo proceso (autoevaluación, coevaluación, etc.).

Anivel didáctico:

- Favorecer los procesos de acogida del alumnado recién llegado dentro de la clase de EF. Como ejemplo se destacan: el acercamiento previo al alumnado recién llegado antes de la clase de EF (presentación del profesorado); el aprendizaje de palabras clave en diferentes idiomas; la realización de actividades de presentación en el aula; y la creación y permanencia de una figura acogedora (compañero/a intérprete) durante las primeras semanas.

- Facilitar la entrada, el acercamiento y la interacción positiva entre el alumnado de diferentes culturas mediante el uso progresivo de agrupaciones culturalmente heterogéneas, usando las agrupaciones libres en las situaciones que se requiera un contexto de confianza y seguridad.

- Utilizar las estrategias comunicativas necesarias para facilitar al alumnado que desconoce la lengua oficial de la escuela, tanto la comprensión de los discursos verbales, como el aprendizaje de este idioma durante las clases prácticas. Algunas de las identificadas en este estudio son: las «parejas lingüísticas» 0 «compañeros/as intérpretes»; la utilización de diferentes idiomas (castellano e inglés); el aprendizaje de palabras clave en los idiomas de los grupos mayoritarios (árabe, tagalo, chino, urdu, etc.); la utilización intencionada del lenguaje gestual; y la reiteración de las demostraciones (información visual).

- Establecer un vínculo afectivo entre el profesorado y el alumnado, donde las relaciones entre ambos se caractericen por la cercanía, el cariño y la calidez.

- Incorporar ejemplos del deporte de élite para reflexionar sobre las conductas racistas o xenófobas que se dan, así como la desigualdad económica existente.

\section{Conclusiones}

El conjunto de intervenciones pedagógicas del profesorado de EF en escuelas multiculturales se caracteriza por su amplia variedad. En este sentido, y como corresponde en una investigación de tipo cualita- 
tivo, se hace patente la imposibilidad de plantear ningún tipo de generalización de los resultados. Este trabajo revela la existencia de tres tendencias en cuanto a esta intervención del profesorado de EF, las cuales han sido tipificadas teóricamente bajo tres tipos de perfiles: el profesorado sensible-inclusor, el profesorado romántico-asimilador y el profesorado quemado-prejuicioso.

Con esta caracterización se pretende ayudar a identificar o reconocer a qué perfil se aproxima más el profesorado de EF para así poder abordar los puntos débiles y reforzar los fuertes, sobre todo en futuras investigaciones o en actividades de formación. Con ello se busca avanzar hacia el necesario futuro de la EF intercultural a partir del conocimiento real de lo que acontece en la escuela y de las percepciones de algunos de sus propios protagonistas, en este caso, del profesorado de EF con experiencia en una escuela multicultural.

\section{Agradecimientos}

Este estudio ha podido ser realizado gracias a la obtención de una beca de investigación predoctoral de cuatro años de duración concedida al Departament d'Expressió Musical, Plàstica i Corporal de la Universitat Autònoma de Barcelona, dentro de la convocatoria de personal investigador en formación (PIF) del curso 2009/2010.

\section{Referencias}

Aguado, M. T. (2003). Pedagogía intercultural. Madrid: McGrawHill.

Aguado, M.T.(Ed.) (2006). Guía Inter: una guía práctica para aplicar la educación intercultural en la escuela. Madrid: Ministerio de Educación y Ciencia. Recuperado de: http://www.uned.es/ grupointer/colentremanos001pc.pdf

Aguado, M. T., Gil, J. A., Jiménez, R. A., Sacristán, A., Ballesteros, B., Malik, B., ... Sánchez, M. F. (1999). En M. T. Aguado (Ed.), Diversidad cultural e igualdad escolar: un modelo para el diagnóstico y desarrollo de actuaciones educativas en contextos escolares multiculturales. Madrid: Ministerio de Educación y Cultura. Centro de publicaciones.

Banks, J. A. (1995). Multicultural education: development, dimensions and challenges. En J. Q. Adams \& J. R. Welsch(Eds.), Multicultural education: Strategies for implementation in colleges and universities. Volume 4 (pp. 3-14). Macomb: Illinois Staff and Curriculum Developers Association.

Bardin, L. (1986). El análisis de contenido. Madrid: Akal.

Bell, N. D. \& Lorenzi, D. (2004). Facilitating second language acquisition in elementary and secondary physical education classes. Journal of Physical Education, Recreationy Dance, 75(6), 46-51.

Besalú, X. (2002). Diversidad cultural y educación. Madrid: Síntesis.

Besalú, X. (2004). La formación inicial en interculturalidad. En J. A. Jordán, X. Besalú, M. Bartolomé, M. T. Aguado, C. Moreno \& M. Sanz(Eds.), La formación del profesoradoeneducación intercultural (pp. 49-91). Madrid: Catarata.

Besalú, X. \& Tort, J. (2009). Escuela y sociedad multicultural: propuestas para trabajar con alumnado extranjero. Alcalá de Guadaira: Mad.

Camacho, H. M. \& Padrón, M. (2006). Malestar docente y formación inicial del profesorado: percepciones del alumnado. Revista Interuniversitaria de Formación del Profesorado, 20(2), 209-230.

Capllonch, M., Godall, T. \& Lleixà, T. (2007). El professorat d'educació física a l'escola multicultural. Percepcions del context i necessitats de formació. Temps d'educacio, (33), 61-74.

Carbonell, F. (2006). L'acollida: acompanyament d'alumnatnouvingut. Vic:Eumo.

Carrasco, E. (2006). Proyecto jóvenes guías: la ayuda entre iguales para incorporar nuevos alumnos. Aula de Innovación Educativa, 153154, 17-19.

Carroll, B. \& Hollinshead, G. (1993). Etnhicity and conflict in physical education. British Educational Journal, 19(1), 59-76.
Contreras, O. (2002). Perspectiva intercultural de la educación física. En T. Lleixà, J. R. Flecha, L. Puigvert, O. Contreras, M. Á. Torralba \& J. Bantulà (Eds.), Multiculturalismo y educación física (pp. 4775). Barcelona: Paidotribo.

Contreras, O., Gil, P., Cecchini, J. A. \& García L, L. M. (2007). Teoría de una educación física intercultural y realidad educativa en España. Paradígma, 28(2), 7-47.

Dagkas, S. (2007). Exploring teaching practices in physical education with culturally diverse classes: a cross-cultural study. European Journal of Teaching Education, 30(4), 431-443.

Essomba, M. Á. (2008). La gestión de la diversidad cultural en la escuela: 10 ideas clave. Barcelona: Graó.

Etxeberría, F. \& Elosegui, K. (2010). Integración del alumnado inmigrante: Obstáculos y propuestas. Revista Española de Educación Comparada, (16), 235-263.

Etxeberría, F., Karrera, I. \& Murua, H. (2010). Competencias interculturales del profesorado con alumnado inmigrante en el País Vasco. Revista Electrónica Interuniversitaria de Formación del Profesorado, 13(4). Recuperado de: http://www.aufop.com/aufop/ uploaded_files/articulos/1291992674.pdf

Flecha, J. R. (2001). Racismo moderno y postmoderno en Europa: enfoque dialógico y pedagogías antirracistas. Revista Española de Investigaciones Sociológicas, (94), 79-103.

Flecha, J. R. \& Puigvert, L. (2002). Multiculturalismo y educación. En T. Lleixà, R. Flecha, L. Puigvert, O. Contreras, M. Á. Torralba \& J. Bantulà (Eds.), Multiculturalismo y educación física (pp. 9-45). Barcelona: Paidotribo.

Flores, G. (2013). El profesorado de educación física ante la realidad multicultural: percepción sobre el alumnado, intervención pedagógica y trayectoria profesional. Un estudio de casos. Tesis Doctoral. Universidad Autónoma de Barcelona, Bellaterra. Recuperado de: http://hdl.handle.net/10803/129186.

Flores, G, Prat, M. \& Soler, S. (2014). La voz del profesorado de educación física sobre su formación académica ante la realidad multicultural: análisis de la situación y propuestas de mejora. Revista Electrónica Interuniversitaria de Formación del Profesorado, 17(2),183-199. Recuperado de: http://www.aufop.com/aufop/ uploaded_files/articulos/1403121378.pdf.

García-Fernández, J. A. \& Goenechea, C. (2009). Educación intercultural: análisis de la situación y propuestas de mejora. Barcelona: Wolters Kluwer España.

Garreta, J. (2011). La atención a la diversidad cultural en Cataluña: exclusión, segregación e interculturalidad. Revista de Educación, (335), 213-233.

Gieß-Stüber, P. (2009). Interculturalidad reflexiva e interacción con lo «extraño» en el deporte y a través del deporte. En J. Durán (Ed.), Actividad física, deporte e inmigración. El reto de la interculturalidad (pp. 95-112). Madrid: Dirección General de Deportes de la Comunidad de Madrid.

Gieß-Stüber, P. \& Kühler, C. (2008). Without the benefit of my studies, I would certainly have put my foot in it... a conversation with a sport teacher. En P. Gieß-Stüber \& D. Blecking (Eds.), Sport Integration-Europe. Widening horizons in intercultural education (pp. 288-294). Baltmannsweiler: Schneider.

Gil-Jaurena, I. (2008). El enfoque intercultural en la educación primaria: una mirada a la práctica escolar. Tesis Doctoral. Universidad Nacional de Educación a Distancia (UNED), Madrid. Recuperado de: http://www.uned.es/grupointer/tesis_ines_gil_jaurena.pdf .

Gil-Madrona, P. \& Pastor, J. C. (2003). Actitudes multiculturales exteriorizadas en educación física: el estudio de un caso y la educación emocional como respuesta. Revista Complutense de Educación, 14(1), 133-158.

Goenechea, C., García-Fernández, J. A. \& Jiménez, R. Á. (2011). Los dilemas de la atención educativa a los alumnos inmigrantes recién llegados: Estudio comparativo de los modelos andaluz (ATAL) y madrileño (aulas de enlace). Profesorado. Revista de Currículumy Formación del Profesorado, 15(3), 263-278. 
Heinemann, K. (2003). Introducción a la metodología de la investigación empírica en las ciencias del deporte ( $1^{\mathrm{a}}$ ed.). Barcelona: Paidotribo.

Jackson, P.W. (1968). Life in classrooms. New York: Holt, Rinehart y Winston

Jordán, J.A. (2004). Introducción: la formación permanente del profesorado en educación intercultural. En J. A. Jordán, X. Besalú, M. Bartolomé, M. T. Aguado Odina, C. Moreno \& M. Sanz (Eds.), La formación del profesorado en educación intercultural (pp. 11-48). Madrid: Catarata.

Jordán, J.A. (2005). ¿Qué educación intercultural para nuestra escuela? Material no publicado. Recuperado de: http:// www.aulaintercultural.org/IMG/pdf/jordan_escuela.pdf .

Latorre, A., Del Rincón, D. \& Arnal, J. (2003). Bases metodológicas de la investigación educativa. Barcelona: Experiencia.

López-Pastor, V. M. (2007). La evaluación en educación física y su relación con la atención a la diversidad del alumnado. Kronos: la Revista Científica de Actividad Física y Deporte, V(11), 59-71.

López-Pastor, V. M., Pérez, Á. \& Monjas, R. (2007). La atención a la diversidad en elárea de educación física. La integración del alumnado con necesidades educativas específicas, especialmente el alumnado inmigrante y de minorías étnicas. Revista digital EFdeportes,(106). Recuperado de: http://www.efdeportes.com/efd106/la-atencion-ala-diversidad-en-educacion-fisica.htm .

López-Rodríguez, F. \& Sentís, F. (2000). Las adaptaciones del currículum en primaria. En R. Alcudia (Ed.), Atención a la diversidad (pp. 85-90). Caracas: Laboratorio Educativo.

Lleixà, T.(2002). Atención a la diversidad en el currículum de educación física. En T. Lleixà, J. R. Flecha, L. Puigvert, O. Contreras, M. Á. Torralba \& J. Bantulà (Eds.), Multiculturalismo y educación física (pp. 79-111). Barcelona: Padiotribo.

Lluch, X. \& Salinas, J. (1996). La diversidad cultural en la práctica educativa: materiales para la formación del profesorado en educación intercultural. Madrid: Ministerio de Educación y Cultura. Centro de publicaciones.

Massot, M. I., Dorio, I. \& Sabariego, M. (2004). Estrategias de recogida y análisis de la información. En R. Bisquerra(Ed.), Metodología de investigación educativa (pp. 329-366). Madrid: La Muralla.

McKernan, J. (1999). Investigación-acción y currículum: métodos y recursos para profesionales reflexivos. Madrid: Morata.

Oliver, M. C. (2003). Estrategias didácticas y organizativas ante la diversidad: dilemas del profesorado. Barcelona: Octaedro.
Oller, C. \& Colomé, E. (2010). Alumnado de otras culturas: acogida y escolarización. Barcelona: Graó.

Ortiz, M. (2008). Inmigración en las aulas: percepciones prejuiciosas de los docentes. Papers, (87), 253-268.

Pastor, J. C., González, S., Cuevas, R. \& Gil-Madrona, P. (2010). El profesorado de educación física ante la inmigración. Cuadernos de Psicología del Deporte, 10(Supl.), 79-84.

Patterson, D. (2012). Building the case for culturally responsive teaching in physical education: using high school students' perceptions toward multiethnic groups. Current Issues in Education, 15(3). Recuperado de: http://cie.asu.edu/ojs/index.php/cieatasu/article/ view/876.

Prat, M. \& Soler, S. (2002). Las posibilidades del juego, la actividad física y el deporte para la mejora de la convivencia. Revista Electrónica Interuniversitaria de Formación del Profesorado, 5(2). Recuperado de: http://www.aufop.com/aufop/uploaded_files/revistas/ 122843928110.pdf .

Prat, M. \& Soler, S. (2003). Actitudes, valores ynormas en la educación física y el deporte: reflexiones y propuestas didácticas. Barcelona: INDE.

Ruiz-Pérez, L.M (2000). Aprender a ser incompetente en educación física: un enfoque psicosocial. Apunts: Educación Física y Deportes, (60), 20-25.

Ruiz-Valdivia, M., Molero, D., Zagalaz, M. L. \& Cachón, J. (2012). Anàlisi de la integració del'alumnat immigrant a través de les classes d'educació física. Apunts. Educació Físicai Esports, 2(108), 26-34.

Sales,A. (2007). La formació dels professionals en educació intercultural. En Grup de Recerca en Educació Intercultural (Ed.), Multiculturalitat, educació i societat (pp. 205-230). Palma de Mallorca: Universitat de les Illes Balears, Edicions UIB.

Soler, S. (2009). Los procesos de reproducción, resistencia y cambio de las relaciones tradicionales de género en la educación física: el caso del fútbol. Cultura y Educación, 21(1), 31-42.

Soler, S., Flores, G \& Prat, M. (2012). La educación física y el deporte como herramientas de inclusión de la población inmigrante en Cataluña: el papel de la escuela y la administración local. Pensar a Prática, 15(1), 1-19.

Torres, J. (2008). Diversidad cultural y contenidos escolares. Revista de Educación, (345), 83-110.

Van Manen, M. (1998). El tacto en la enseñanza. El significado de la sensibilidad pedagógica. Barcelona: Paidós.

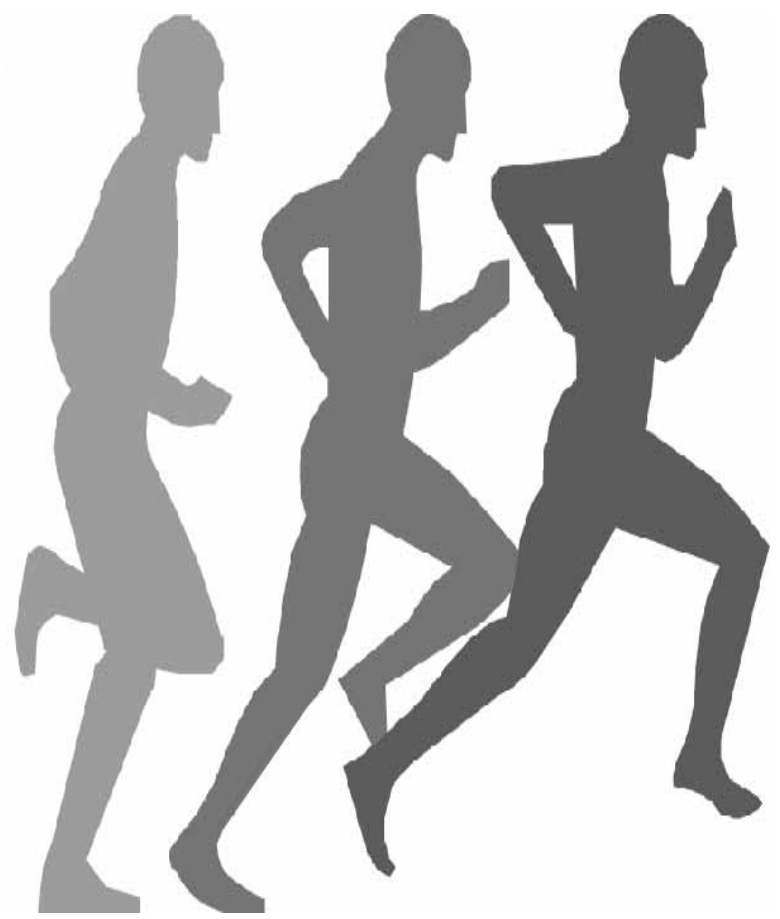

